\title{
Quantum of the Magnetic Flux Characteristic for Experiments Performed on the Integer and Fractional Quantum Hall Effects
}

\author{
Stanisław Olszewski \\ Institute of Physical Chemistry, Polish Academy of Sciences, Warsaw, Poland \\ Email: olsz@ichf.edu.pl
}

Received August 28, 2013; revised September 27, 2013; accepted October 26, 2013

Copyright (C) 2013 Stanisław Olszewski. This is an open access article distributed under the Creative Commons Attribution License, which permits unrestricted use, distribution, and reproduction in any medium, provided the original work is properly cited.

\begin{abstract}
Experimentally the plateaus characteristic for the integer quantum Hall effect is obtained in vicinity of specific values of the magnetic induction. The paper demonstrates that the ratios of these induction values to carrier concentration in the planar crystalline samples approach systematically the quanta of the magnetic flux important for the behavior of superconductors. Moreover, the same quanta can be deduced from the Landau levels theory and their application in the magnetoresistance theory gives results being in accordance with experiments. The quanta of the magnetic flux similar to those for the integer quantum Hall effect can be obtained also for the fractional quantum Hall effect. This holds on condition the experimental ratio of the magnetic flux to carrier concentration is multiplied by the filling factor of the Landau level.
\end{abstract}

Keywords: Quantum Hall Effect-Integer and Fractional; Magnetic Induction; Carrier Concentration; Quanta of the Orbital Magnetic Flux

\section{Introduction}

Experimentally the discovery of the integer quantum Hall effect for the planar metallic-like crystals led to a result that the magnetic flux in such crystals is quantized with a very high accuracy by the values equal to

$$
\Phi=\frac{h c}{e^{*}}
$$

determined from the Bohr-Sommerfeld quantization condition imposed on the orbital motion of a charged particle in the magnetic field (see e.g. [1]). Equation (1) gives a tool in obtaining the effective charge $e^{*}$ of that particle. A well-known result for $\Phi$ in case of the superconducting systems is $[2,3]$

$$
\Phi=\frac{h c}{2 e}=2.07 \times 10^{-7} \text { gauss } \times \mathrm{cm}^{2} .
$$

Result in (2) implies that the effective charge carried in superconductors is a double of the electron charge, i.e.

$$
e^{*}=2 e,
$$

leading to an idea of the coupled electron pairs as the particles providing the electric current. More recently, a source of interest in $\Phi$ is raised by the fractional quantum Hall effect in which $e^{*}$ is considered as a fraction of e $[4,5]$.

Our aim is to examine the $\Phi$ in the quantum Hall effect beginning with the integer form of that effect:

$$
\frac{h}{e^{2}} \text {. }
$$

In Section 2, we show that the flux in (2) leads to the result presented in (4).

The formula of (4) is characteristic for the magnetoresistance of the integer quantum Hall effect, but also the magnetoresistance values

$$
\frac{h}{v e^{2}}
$$

specific for the fractional quantum Hall effect can be obtained [6-8]. Here the coefficient $v$ is a simple fraction like

$$
\frac{1}{3}, \frac{2}{3}, \frac{2}{5}, \cdots
$$

A predominant feature of (6) is that any fraction has an odd integer number in its denominator. 
As a rule the experiments leading to (4)-(6) were performed for some definite value of the magnetic field induction $B_{z}$, or some plateau of these values. The $B_{z}$ directed, say, parallelly to the axis $z$ were assumed to be normal to the planar crystalline samples, so each experiment concerned a specific concentration $n$ of the electric carriers (electrons or holes) present in the sample plane.

Theoretically an approach to the quanta of (4) and (5) can be attained by calculating the magnetoresistance of a metal on the basis of a single free-electron like band of states [9-12]. Here the off-diagonal Hall resistance and diagonal resistance can be considered.The change $\Delta \rho_{x x}$ of the diagonal tensor component of the electric resistance $\rho_{x x}$ upon the action of the magnetic field is represented by the formula

$$
\Delta \rho_{x x}=\frac{\Delta \rho_{x x}}{\rho_{x x}} \rho_{x x}=\frac{\tau_{\mathrm{el}} \Omega_{0}}{\xi} \frac{m}{n e^{2} \tau_{\mathrm{el}}} .
$$

Expression (7) holds because [9-12]

$$
\frac{\Delta \rho_{x x}}{\rho_{x x}}=\frac{\tau_{\mathrm{el}}}{\tau_{\operatorname{mag}}}=\frac{\tau_{\mathrm{el}} \Omega_{0}}{\xi}
$$

and

$$
\rho_{x x}=\frac{m}{n e^{2} \tau_{\mathrm{el}}} ;
$$

see e.g. [13]. The $\tau_{\mathrm{el}}$ is the relaxation time for the electric resistance,

$$
\Omega_{0}=\frac{e B_{z}}{m c}
$$

is the electron gyration frequency in the field $B_{z}$,

$$
\tau_{\text {mag }}=\frac{\xi}{\Omega_{0}}
$$

is the relaxation time due to the presence of the magnetic field,

$$
\xi=\frac{1}{2}
$$

is a constant number entering formula (11) obtained on the basis of a quantum-mechanical calculation $[9,11]$. Because of (12) we obtain from (7):

$$
\Delta \rho_{x x}=\frac{2 B_{z}}{n e c} .
$$

Expression (13) is equal to the double absolute value of the Hall resistance:

$$
\left|\rho_{x y}\right|=\frac{B_{z}}{n e c}
$$

The formula (14) remains unmodified in comparison with the well-known result obtained before (see e.g. [14]) without any use of $\tau_{\text {mag }}$. The lack of change is due to the property of the tensor which is representing the modification of the electric resistance given by the presence of the magnetic field: the tensor contains $\tau_{\text {mag }}$ both in the numerator and denominator of the off-diagonal matrix element representing the Hall effect, so [9,11]:

$$
\begin{aligned}
\left|\Delta \rho_{x y}\right| & =\frac{m \xi}{n e^{2} \tau_{\mathrm{mag}}}=\frac{m \Omega_{0} \tau_{\mathrm{mag}}}{n e^{2} \tau_{\mathrm{mag}}} \\
& =\frac{m \Omega_{0}}{n e^{2}}=\frac{B_{z}}{n e c}=\left|\rho_{x y}\right|
\end{aligned}
$$

on condition we note that

$$
\xi=\tau_{\operatorname{mag}} \Omega_{0}
$$

because of the formula (11) for $\xi$. In the last step of (15), a conventional notation for the Hall resistance is applied. A similar property of independence on the relaxation time has the Hall matrix element of the original tensor for the magnetoresistance [13], but this concerns the relaxation time $\tau_{\mathrm{el}}$ due to the electric field and not $\tau_{\operatorname{mag}}$ for the magnetic field.

The plateaus of magnetoresistance which signalize the presence of the integer quantum Hall effect refer to some definite experimental values of $B_{z}$ and $n$. Because of the ratio

$$
B_{z} / n
$$

entering (13), (14) and (15) our aim is to examine (17) in some detail.

\section{Electron Population of the Landau Levels Provides Us with the Quanta of Magnetoresistance and Those of the Magnetic Flux}

For the magnetic field $B_{z}$ strong enough to collect all electrons on a single Landau level, the number of electron orbits occupying that level is equal to [14]

$$
D=\frac{e L^{2}}{2 \pi \hbar c} B_{z}=\frac{n}{2} L^{2} .
$$

In the second step of (18) the spin degeneracy of the orbits is explicitly taken into account, $L$ is the edge of the square occupied by the planar metallic-like sample. In a situation represented by (18) the ratio (17) becomes:

$$
\frac{B_{z}}{n}=\frac{\pi \hbar c}{e}=\frac{1}{2} \frac{h c}{e}
$$

which is identical with (2); see also [13]. The ratio of (19) can be substituted to the formulae (13) and (14)-(15). In the first case the result

$$
\Delta \rho_{x x}=\frac{2}{e c} \frac{1}{2} \frac{h c}{e}=\frac{h}{e^{2}}
$$

is identical to (4); in the second case we obtain 


$$
\left|\rho_{x y}\right|=\frac{1}{e c} \frac{1}{2} \frac{h c}{e}=\frac{1}{2} \frac{h}{e^{2}}
$$

which is a half of the diagonal magnetoresistance in (20). Henceforth we consider (21) as a quantum of the Hall resistance given by a fully occupied single Landau level.

An application of the formalism can be done for a textbook example of the experimental presentations of $\Delta \rho_{x x}$ and $\rho_{x y}$ for which, however, no carrier concentration $n$ for the examined two-dimensional system has been given [15]. The obtacle can be avoided when, in the first step, the equivalence of (14) and (21) for a completely filled Landau level is taken into account. In this case

$$
\frac{B_{z}}{n e c}=\frac{1}{2} \frac{h}{e^{2}}
$$

so

$$
n=\frac{2 B_{z} e}{h c} .
$$

Experimentally the quantum Hall plateau corresponding to the Hall resistance (21) begins at $B_{z}=4 \mathrm{~T}=4 \times 10^{4}$ gauss. Therefore from (23)

$$
\begin{aligned}
n & =\frac{8 \times 10^{4} \times 4.803 \times 10^{-10}}{6.62 \times 10^{-27} \times 3 \times 10^{10}} \\
& =1.93 \times 10^{11} \mathrm{~cm}^{2} .
\end{aligned}
$$

This $n$ provides us with the ratio

$$
\begin{aligned}
\frac{B_{z}}{n} & =\frac{4 \times 10^{4}}{1.93 \times 10^{11}} \\
& =2.07 \times 10^{-7} \text { gauss } \times \mathrm{cm}^{2}
\end{aligned}
$$

being in a perfect agreeement with (19); see (2).

The end point of the plateau is at $B_{z} \approx 7 \mathrm{~T}$ and this gives the magnetic flux per electron

$$
\begin{aligned}
\frac{B_{z}}{n} & =\frac{7 \times 10^{4}}{1.93 \times 10^{11}} \\
& =3.6 \times 10^{-7} \text { gauss } \times \mathrm{cm}^{2},
\end{aligned}
$$

therefore dispersion of $\Phi$ for a given $n$ can be large.

\section{Calculation of the Magnetic Flux from the Experimental Data for $B_{z}$ and $n$}

Rather surprisingly the ratio (17) leading to the quantum (21) of the Hall resistance seems to be not very extensively examined on the experimental basis. In Table 1 we present a list of the values of $B_{z}, n$ and ratios $B_{z} / n$ associated with the plateaus of the quantum Hall resistance observed in different compounds; see [16-41]. This study shows that the experimental data for $B_{z} / n$ approach the quantum value obtained for (2) and in (19).

\begin{tabular}{|c|c|c|c|}
\hline Reference & $\begin{array}{c}B_{z} \\
\text { (in T) } \\
\end{array}$ & $\begin{array}{c}n \\
\text { (in } 10^{11} \mathrm{~cm}^{-2} \text { ) }\end{array}$ & $\begin{array}{c}B_{z} / n \\
\text { (in } 10^{-7} \text { gauss } \times \mathrm{cm}^{2} \text { ) }\end{array}$ \\
\hline$[16]$ & 15 & 7.8 & 1.92 \\
\hline [17] & 5 & 2.46 & 2.03 \\
\hline [18] & 8 & 4.0 & 2.0 \\
\hline [19] & 3 & 1.48 & 2.03 \\
\hline$[20]$ & 2.5 & 1.23 & 2.03 \\
\hline$[21]$ & 4.3 & 2.1 & 2.05 \\
\hline$[22]$ & 6.7 & 3.4 & 1.8 \\
\hline [23] & 4.0 & 1.5 & 2.67 \\
\hline [24] & 4 & 2.1 & 1.9 \\
\hline$[24]$ & 6.5 & 3.5 & 1.9 \\
\hline [25] & 6.0 & 3.0 & 2.0 \\
\hline$[26]$ & 5.2 & 2.23 & 2.26 \\
\hline [27] & 9.0 & 6.0 & 1.5 \\
\hline [28] & $1 / 2 \times 6.0$ & 1.45 & 2.07 \\
\hline [29] & 5.0 & 2.4 & 2.08 \\
\hline [30] & $2 \times 5.4$ & 5.6 & 1.93 \\
\hline$[30]$ & $3 \times 3.8$ & 5.6 & 2.04 \\
\hline$[30]$ & $4 \times 2.8$ & 5.6 & 2.0 \\
\hline [30] & $5 \times 2.2$ & 5.6 & 1.96 \\
\hline [31] & 0.8 & 0.4 & 2.0 \\
\hline [32] & 2.0 & 1.04 & 1.92 \\
\hline [33] & 1.2 & 0.571 & 2.1 \\
\hline [34] & 1.5 & 0.581 & 2.58 \\
\hline$[35]$ & 2.25 & 1.06 & 2.12 \\
\hline [36] & 10 & 4 & 2.5 \\
\hline [37] & 2.7 & 1.26 & 2.14 \\
\hline$[38]$ & 1.5 & 0.66 & 2.27 \\
\hline [38] & 0.9 & 0.41 & 2.2 \\
\hline$[38]$ & 2.5 & 1.26 & 1.98 \\
\hline [39] & 4.3 & 1.93 & 2.2 \\
\hline [39] & 6.0 & 3.0 & 2.0 \\
\hline$[40]$ & 3.2 & 1.53 & 2.09 \\
\hline$[41]$ & 10.5 & 5.1 & 2.06 \\
\hline$[41]$ & 7.9 & 3.8 & 2.08 \\
\hline [41] & 5.5 & 2.65 & 2.08 \\
\hline
\end{tabular}
The dispersion of the data in Table $\mathbf{1}$ is not much diffe-
Table 1. List of the experimental $B_{z}$ values of the magnetic induction (in the tesla units) connected with the plateaus of the quantum integer Hall effect [equal to $(1 / 2) h / e^{2}$, carrier concentrations $n$ (in $10^{11} \mathrm{~cm}^{-2}$ ) and the ratios $B_{z} / n$. In some cases the data are observed for other Landau levels than the first one and transformed to those corresponding to a single fully occupied Landau level. 
rent from that observed in superconducting cyllinders [42].

For some experiments the ratio

$$
\frac{\left|\rho_{x y}\right|}{\Delta \rho_{x x}}=\frac{1}{2}
$$

[see (20) and (21)] could find its confirmation in the observed data obtained for almost the same induction value $B_{z}[29,39]$.

\section{Discussion: Reference to the Fractional Quantum Hall Effect}

A natural extension of the problem of $\Phi$ considered for the integer quantum Hall effect concerns the fractional version of that effect. In this case the orbit population (18) a Landau level is changed by a factor of $v$ giving the relation

$$
v \frac{e B_{z}}{h c}=\frac{n}{2} .
$$

Evidently in this case we obtain the formula

$$
v \frac{B_{z}}{n}=\frac{1}{2} \frac{h c}{e}
$$

instead of (19), so the expected magnetic flux per one carrier unit becomes

$$
\Phi=\frac{B_{z}}{n}=\frac{h c}{e^{*}}=\frac{h c}{2 e v} .
$$

Here it should be noted that only a substitution of $B_{z} / n$ from the formula (29) into (13) can provide us with the result

$$
\Delta \rho_{x x}=\frac{2}{e c} \frac{h c}{2 e v}=\frac{h}{e^{2} v}
$$

in accordance with experiment; see (5). Since we have mainly $v<1$, the formula (29) implies the presence of the carriers having their charge

$$
e^{*}=2 e v<2 e .
$$

However, an alternative approach to $\Phi$ can be obtained on the basis of the assumption that no $e^{*}<2 e$ are present but the number of the filled Landau levels which take part in experiment is increased from 1 to $1 / v$; for the sake of simplicity we assume that $1 / v$ is an integer number [43]. An increase of the filled levels number can be attained by a corresponding change of $B_{z}$ for a single filled level to $v B_{z}$ which makes on each level a decrease of the original concentration $n$ to $v n$. In effect we obtain

$$
\Phi_{L}=\frac{v B_{z}}{v n}=\frac{B_{z}}{n}=\frac{1}{2} \frac{h c}{e}
$$

for each component level which is the same ratio as for a single filled Landau level; see (19). In result of that situation only a sum of $\Phi_{L}$ in (32) performed over all occupied $1 / v$ levels can give the magnetic flux equal to that presented in (29).

Since $v$ is a constant and $n$ is a discrete integer parameter, the behaviour of the magnetic flux in the quantum Hall effects indicates that $B_{z}$ in (29) should be a quantized parameter. The quantum of $e B_{z}$ is expected to be the smallest experimentally acceptable number of

$$
\frac{n}{2 v} h c \sim 10^{-16} \text { dyne }
$$

obtained on condition we assume that $n \sim v \sim 1$.

By considering $v B_{z} / n$ as the magnetic flux $\Phi$ it can be noted that the following relation exists between $\Phi$, the energy $E$ of the quantum state and current $j=n e v_{x}[44]$ :

$$
j=\alpha \frac{\partial E}{\partial \Phi}
$$

where $\alpha$ is a constant. In fact, for a one electron energy on the Landau level obtained by the change of $B_{z}$ to $v B_{z}$, viz.

$$
E=v N_{L} \hbar \Omega_{0},
$$

we obtain from (34) the relation:

$$
\begin{aligned}
n e v_{x} & =n e \frac{\hbar k_{x}^{F}}{m}=\alpha \frac{\partial\left(v N_{L} \hbar \Omega_{0}\right)}{\partial\left(v B_{z} / n\right)} \\
& =\alpha \frac{\partial\left(N_{L} \hbar e B_{z} n\right)}{\partial\left(m c B_{z}\right)}=\alpha \frac{N_{L} \hbar e n}{m c}
\end{aligned}
$$

which gives

$$
\alpha=\frac{c k_{x}^{F}}{N_{L}},
$$

representing a number independent of $v, B_{z}$ and $n$. The $k_{x}^{F}$ is a component of the Fermi wave vector, $N_{L}$ is the index of the Landau level.

\section{Examples of the Magnetic Flux Calculated for the Fractional Quantum Hall Effect}

In Tables 2-4, we present several examples of the quanta of the magnetic flux obtained when the measured data for $B_{z}, n$ and $v$ entering the experiments on the fractional quantum Hall effect are substituted on the left-hand side of the formula (28). These quanta approach evidently the result given in (2), as it could be expected on the basis of (28). Similar quanta of Table 1 are, in average, more distant from the result in (2) because the very existence of plateaus of $B_{z}$ in the integer quantum Hall effect makes the calculations of $\Phi$ more uncertain than in 
Table 2. Quanta of the magnetic flux $\frac{v B_{z}}{n}$ for the fractional quantum Hall effect deduced from the experimental data corresponding to a similar set of $v$ but different carrier densities $n$ are taken into account. See [38], Figure 9.15 and [37]. The factor of 2 in $n$ is due to spin degeneracy.

\begin{tabular}{|c|c|c|c|}
\hline$v$ & $\begin{array}{c}\quad B_{z} \\
\text { (in tesla units) }\end{array}$ & $\begin{array}{c}n \\
\text { (in } 10^{11} \mathrm{~cm}^{-2} \text { ) }\end{array}$ & $\begin{array}{c}\frac{v B_{z}}{n} \\
\left(\text { in } 10^{-7} \text { gauss } \times \mathrm{cm}^{2} \text { ) }\right.\end{array}$ \\
\hline $2 / 3$ & 14.25 & $2 \times 0.68$ & 2.08 \\
\hline $3 / 5$ & 14.75 & $2 \times 0.68$ & 2.09 \\
\hline $1 / 2$ & 15.75 & $2 \times 0.68$ & 2.11 \\
\hline $2 / 5$ & 17.75 & $2 \times 0.68$ & 2.06 \\
\hline $1 / 3$ & 18.75 & $2 \times 0.68$ & 1.96 \\
\hline $1 / 5$ & 13.75 & $2 \times 0.68$ & 1.91 \\
\hline $2 / 3$ & 15.75 & $2 \times 0.92$ & 2.08 \\
\hline $3 / 5$ & 16.25 & $2 \times 0.92$ & 2.04 \\
\hline $1 / 2$ & 17.52 & $2 \times 0.92$ & 2.04 \\
\hline $2 / 5$ & 19.52 & $2 \times 0.92$ & 2.07 \\
\hline $1 / 3$ & 11.75 & $2 \times 0.92$ & 1.99 \\
\hline $2 / 3$ & 16.25 & $2 \times 1.03$ & 2.02 \\
\hline $3 / 5$ & 17.25 & $2 \times 1.03$ & 2.04 \\
\hline $1 / 2$ & 18.52 & $2 \times 1.03$ & 2.06 \\
\hline $2 / 5$ & 10.52 & $2 \times 1.03$ & 2.04 \\
\hline $1 / 3$ & 12.52 & $2 \times 1.03$ & 2.02 \\
\hline $2 / 3$ & 16.75 & $2 \times 1.10$ & 2.05 \\
\hline $3 / 5$ & 17.52 & $2 \times 1.10$ & 2.05 \\
\hline $1 / 2$ & 19.75 & $2 \times 1.10$ & 2.05 \\
\hline $2 / 5$ & 11.25 & $2 \times 1.10$ & 2.05 \\
\hline $1 / 3$ & 13.52 & $2 \times 1.10$ & 2.05 \\
\hline $4 / 5$ & 16.5 & $2 \times 1.16$ & 2.07 \\
\hline $2 / 3$ & 17.5 & $2 \times 1.16$ & 2.01 \\
\hline $3 / 5$ & 18.5 & $2 \times 1.16$ & 2.07 \\
\hline $1 / 2$ & 19.5 & $2 \times 1.16$ & 2.05 \\
\hline $1 / 3$ & 14.5 & $2 \times 1.16$ & 2.08 \\
\hline $4 / 5$ & 17 & $2 \times 1.32$ & 2.12 \\
\hline $2 / 3$ & 18 & $2 \times 1.32$ & 2.02 \\
\hline $1 / 2$ & 11 & $2 \times 1.32$ & 2.08 \\
\hline $4 / 5$ & 17.5 & $2 \times 1.43$ & 2.10 \\
\hline $2 / 3$ & 19.5 & $2 \times 1.43$ & 2.10 \\
\hline $4 / 7$ & 10.5 & $2 \times 1.43$ & 2.10 \\
\hline $1 / 2$ & 11.5 & $2 \times 1.43$ & 2.01 \\
\hline $4 / 5$ & 8.5 & $2 \times 1.55$ & 2.06 \\
\hline $2 / 3$ & 9.5 & $2 \times 1.55$ & 2.04 \\
\hline $4 / 7$ & 11.5 & $2 \times 1.55$ & 2.03 \\
\hline $1 / 2$ & 13.5 & $2 \times 1.55$ & 2.09 \\
\hline
\end{tabular}

Table 3. List of the experimental $B_{z}$ values of the magnetic induction (in the tesla units) connected with the magnetoresistance maxima due to the fractional quantum Hall effect observed for different filling factors $v$ and carrier concentration $n$ (in $10^{11} \mathrm{~cm}^{-2}$ ) in different crystal samples [28]. The ratios $v B_{z} / n$ are calculated in $10^{-7}$ gauss $\times \mathrm{cm}^{2}$ units. The $n$ of [28] listed in the Table are doubled because of the spin degeneracy taken into account.

\begin{tabular}{cccc}
\hline$v$ & $B_{z}$ & $n$ & $\begin{array}{c}v B_{z} / n \\
\end{array}$ \\
(in T) & $\left(\right.$ in $\left.10^{11} \mathrm{~cm}^{-2}\right)$ & $\left(\right.$ in $10^{-7}$ gauss $\left.\times \mathrm{cm}^{2}\right)$ \\
\hline $1 / 3$ & 19 & $2 \times 1.53$ & 2.07 \\
$2 / 3$ & 15 & $2 \times 2.42$ & 2.07 \\
$2 / 5$ & 22 & $2 \times 2.13$ & 2.07 \\
$3 / 5$ & 14.7 & $2 \times 2.13$ & 2.07 \\
$5 / 3$ & 5.3 & $2 \times 2.06$ & 2.14 \\
$3 / 7$ & 20.6 & $2 \times 2.13$ & 2.07 \\
\hline
\end{tabular}

Table 4. The magnetic flux calculated from the experimental data for $v, B_{z}$ and $n$ given in the diagrams of [45].

\begin{tabular}{cccc}
\hline$v$ & $B_{z}$ & $n$ & $v B_{z} / n$ \\
& (in tesla) & $\left(\right.$ in $\left.10^{11} \mathrm{~cm}^{-2}\right)$ & $\left(\right.$ in $10^{-7}$ gauss $\left.\times \mathrm{cm}^{2}\right)$ \\
\hline 2 & 15.3 & $2 \times 2.6$ & 2.04 \\
$8 / 5$ & 16.8 & $2 \times 2.6$ & 2.09 \\
$10 / 7$ & 17.4 & $2 \times 2.6$ & 2.03 \\
$4 / 3$ & 18.2 & $2 \times 2.6$ & 2.10 \\
$6 / 5$ & 18.9 & $2 \times 2.6$ & 2.05 \\
1 & 10.8 & $2 \times 2.6$ & 2.08 \\
$4 / 5$ & 13.2 & $2 \times 2.6$ & 2.00 \\
$2 / 3$ & 16.2 & $2 \times 2.6$ & 2.05 \\
$4 / 7$ & 18.2 & $2 \times 2.6$ & 1.98 \\
$1 / 2$ & 21.2 & $2 \times 2.6$ & 2.02 \\
$4 / 9$ & 23.2 & $2 \times 2.6$ & 1.97 \\
$2 / 5$ & 27.2 & $2 \times 2.6$ & 2.08 \\
$1 / 3$ & 33.2 & $2 \times 2.6$ & 2.12 \\
\hline
\end{tabular}

case of the fractional quantum Hall effect.

\section{REFERENCES}

[1] J. C. Slater, "Quantum Theory of Molecules and Solids," Vol. 3, McGraw-Hill, New York, 1967.

[2] J. M. Ziman, "Principles of the Theory of Solids," Cambridge University Press, 1972. http://dx.doi.org/10.1017/CBO9781139644075

[3] M. Cyrot and R. Pavuna, "Introduction to Superconductivity and High $T_{c}$ Materials," World Scientific, Singapore, 1992.

[4] S. A. Kivelson and V. L. Pokrovsky, Physical Review B, 
Vol. 40, 1989, p. 1373. http://dx.doi.org/10.1103/PhysRevB.40.1373

[5] J. K. Jain, S. A. Kivelson and D. J. Thouless, Physical Review Letters, Vol. 71, 1993, p. 3003. http://dx.doi.org/10.1103/PhysRevLett.71.3003

[6] A. H. MacDonald, "Quantum Hall Effect: A Perspective," Kluwer, Milano, 1989. http://dx.doi.org/10.1007/978-94-010-9709-3

[7] R. E. Prange and S. M. Girvin, "The Quantum Hall Effect," 2nd Edition, Springer, New York, 1990. http://dx.doi.org/10.1007/978-1-4612-3350-3

[8] S. Das Sarma and A. Pinczuk, "Perspectives in Quantum Hall Effects,” Wiley, New York, 1997.

[9] S. Olszewski and M. Gluzinski, Zeitschrift für Naturforschung A, Vol. 66, 2011, p. 311.

[10] S. Olszewski, Acta Physica Polonica A, Vol. 120, 2011, p. 525.

[11] S. Olszewski, Reviews in Theoretical Science, Vol. 1, 2013, p. 344. http://dx.doi.org/10.1166/rits.2013.1011

[12] S. Olszewski, Quantum Matter, Vol. 2, 2013, p. 102. http://dx.doi.org/10.1166/qm.2013.1030

[13] C. Kittel, "Quantum Theory of Solids," 2nd Edition, Wiley, New York, 1987.

[14] C. Kittel, "Introduction to Solid State Physics," 7th Edition, Wiley, New York, 1996.

[15] J. Singleton, "Band Theory and Electronic Properties of Solids," Oxford University Press, Oxford, 2001.

[16] Th. Englert, D. C. Tsui, A. C. Gossard and Ch. Uihlein, Surface Science, Vol. 113, 1982, p. 295. http://dx.doi.org/10.1016/0039-6028(82)90604-5

[17] D. C. Tsui, H. L. Stormer and A. C. Gossard, Physical Review Letters, Vol. 48, 1982, p. 1559. http://dx.doi.org/10.1103/PhysRevLett.48.1559

[18] M. A. Paalanen, D. C. Tsui and A. C. Gossard, Physical Review B, Vol. 25, 1982, p. 5566. http://dx.doi.org/10.1103/PhysRevB.25.5566

[19] D. C. Tsui, H. L. Stormer, J. C. M. Hwang, J. S. Brooks and M. J. Naughton, Physical Review B, Vol. 28, 1983, p. 2274. http://dx.doi.org/10.1103/PhysRevB.28.2274

[20] H. L. Stormer, D. C. Tsui, A. C. Gossard and J. C. M. Hwang, Physica B, Vol. 117/118, 1983, p. 688. http://dx.doi.org/10.1016/0378-4363(83)90624-1

[21] A. M. Chang, P. Berglund, D. C. Tsui, H. L. Stormer and J. C. M. Hwang, Physical Review Letters, Vol. 53, 1984, p. 997. http://dx.doi.org/10.1103/PhysRevLett.53.997

[22] H. P. Wei, D. C. Tsui and A. M. M. Pruisken, Physical Review B, Vol. 33, 1985, p. 1488. http://dx.doi.org/10.1103/PhysRevB.33.1488

[23] G. S. Boebinger, A. M. Chang, H. L. Stormer and D. C. Tsui, Physical Review B, Vol. 32, 1985, p. 4268. http://dx.doi.org/10.1103/PhysRevB.32.4268

[24] R. J. Clark, R. J. Nicholas, A. Usher, C. T. Foxon and J. J. Harris, Surface Science, Vol. 170, 1986, p. 141. http://dx.doi.org/10.1016/0039-6028(86)90953-2

[25] R. L. Willett, J. P. Eisenstein, H. L. Stormer, D. C. Tsui,
A. C. Gossard and J. H. English, Physical Review Letters, Vol. 59, 1987, p. 1776.

http://dx.doi.org/10.1103/PhysRevLett.59.1776

[26] J. P. Eisenstein, H. L. Stormer, L. Pfeiffer, and K. W. West, Physical Review Letters, Vol. 62, 1989, p. 1540. http://dx.doi.org/10.1103/PhysRevLett.62.1540

[27] G. S. Boebinger, H. W. Jiang, L. N. Pfeiffer and K. W. West, Physical Review Letters, Vol. 64, 1990, p. 1793. http://dx.doi.org/10.1103/PhysRevLett.64.1793

[28] A. M. Chang, "The Quantum Hall Effect," In: R. E. Prange and S. M. Girvin, Eds., The Quantum Hall Effect, Chapter 6, 2nd Edition, Springer, New York, 1990. http://dx.doi.org/10.1007/978-1-4612-3350-3

[29] J. P. Eisenstein and H. L. Stormer, Science, Vol. 248, 1990, p. 1510 http://dx.doi.org/10.1126/science.248.4962.1510

[30] M. E. Cage in "The Quantum Hall Effect," In: R. E. Prange and S. M. Girvin, Eds., The Quantum Hall Effect, Chapter 2, 2nd Edition, Springer, New York, 1990. http://dx.doi.org/10.1007/978-1-4612-3350-3

[31] M. B. Santos, Y. W. Suen, M. Shayegan, Y. P. Li, L. W. Engel and D. C. Tsui, Physical Review Letters, Vol. 68, 1992, p. 1188.

http://dx.doi.org/10.1103/PhysRevLett.68.1188

[32] J. P. Eisenstein, G. S. Boebinger, L. N. Pfeiffer, K. W. West and Song He, Physical Review Letters, Vol. 68, 1992, p. 1383. http://dx.doi.org/10.1103/PhysRevLett.68.1383

[33] V. Goldman, J. K. Wang, B. Su and M. Shayegan, Physical Review Letters, Vol. 70, 1993, p. 647. http://dx.doi.org/10.1103/PhysRevLett.70.647

[34] T. Sajoto, Y. P. Li, L. W. Engel, D. C. Tsui and M. Shayegan, Physical Review Letters, Vol. 70, 2321, 1993. http://dx.doi.org/10.1103/PhysRevLett.70.2321

[35] H. C. Manoharan and M. Shayegan, Physical Review B, Vol. 50, 1994, p. 17662. http://dx.doi.org/10.1103/PhysRevB.50.17662

[36] P. T. Coleridge, A. S. Sachrajda, H. Lafontaine and Y. Feng, Physical Review B, Vol. 54, 1996, p. 14518. http://dx.doi.org/10.1103/PhysRevB.54.14518

[37] H. C. Manoharan, Y. W. Suen, M. B. Santos and M. Shayegan, Physical Review Letters, Vol. 77, 1996, p. 1813. http://dx.doi.org/10.1103/PhysRevLett.77.1813

[38] M. Shayegan, "Perspectives of Quantum Hall Effects," In: S. Das Sarma and A. Pinczuk, Eds., Perspectives in Quantum Hall Effects, Chapter 9, Wiley, New York, 1997.

[39] D. C. Tsui, Reviews of Modern Physics, Vol. 71, 1999, p. 891. http://dx.doi.org/10.1103/RevModPhys.71.891

[40] C. T. Liang, C. G. Smith, M. Y. Simmons and D. A. Ritchie, Physical Review B, Vol. 64, 2001, Article 233319. http://dx.doi.org/10.1103/PhysRevB.64.233319

[41] K. Vyborny, L. Smrcka and R. A. Deutschmann, Physical Review B, Vol. 66, 2002, Article 205318. http://dx.doi.org/10.1103/PhysRevB.66.205318

[42] B. S. Deaver and W. M. Fairbank, Physical Review Letters, Vol. 7, 1961, p. 43.

http://dx.doi.org/10.1103/PhysRevLett.7.43 
[43] S. Olszewski, Quantum Matter, in press.

[44] H. Aoki, Reports on Progress in Physics, Vol. 80, 1987, p. 655. http://dx.doi.org/10.1088/0034-4885/50/6/002
[45] T. M. Lu, W. Pan, D. C. Tsui, C.-H. Lee, and C. W. Liu, Physical Review B, Vol. 85, 2012, Article 121307. 